\title{
Trilateration based localization method using mobile anchor in wireless sensor networks
}

\author{
M. G. Kavitha' ${ }^{1}$, K. Vinoth Kumar ${ }^{2}$,T. Jayasankar ${ }^{3}$ \\ ${ }^{1}$ Department of CSE, University College of Engineering, India \\ ${ }^{2}$ Department of ECE, SSM Institute of Engineering and Technology, India \\ ${ }^{3}$ Department of ECE, University College of Engineering, India
}

\begin{tabular}{l} 
Article Info \\
\hline Article history: \\
Received Apr 23, 2019 \\
Revised Oct 25, 2019 \\
Accepted Dec 20, 2019 \\
\hline
\end{tabular}

\section{Keywords:}

Localization

LUMAT

Mobile anchor nodes

Wireless sensor networks

\begin{abstract}
Localization in wireless sensor networks (WSNs) is essential in many applications like target tracking, military applications and environmental monitoring. Anchors which are equipped with global positioning system (GPS) facility are useful for finding the location information of nodes. These anchor nodes may be static or dynamic in nature. In this paper, we propose mobile anchors assisted localization algorithm based on regular hexagons in two-dimensional WSNs. We draw a conclusion that the number of anchor nodes greatly affect the performance of localization in a WSN. An optimal number of anchor nodes significantly reduces the localization error of unknown nodes and also guarantees that unknown nodes can obtain high localization accuracy. Because of the mobility of anchor nodes high volume of sensing region is covered with less period of time and hence the coverage ratio of the proposed algorithm increases. Number of communications also decreases for the reason that the system contains $\log _{e}(n)$ number of anchor nodes which leads to less energy consumption at nodes. Simulation results show that our LUMAT algorithm significantly outperforms the localization method containing single anchor node in the network. Movement trajectories of mobile anchors should be designed dynamically or partially according to the observable environment or deployment situations to make full use of realtime information during localization. This is the future research issue in the area of mobile anchor assisted localization algorithm.
\end{abstract}

This is an open access article under the CC BY-SA license.

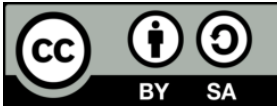

\section{Corresponding Author:}

K. Vinoth Kumar,

Department of ECE,

SSM Institute of Engineering and Technology,

Dindigul, Tamil Nadu, India-624 002.

Email: vinodkumaran87@gmail.com

\section{INTRODUCTION}

A sensor network comprises of a large number of sensor nodes that are densely deployed in a field. Each sensor performs sensing task for detecting specific events. The sink node is responsible for collecting sensed data reported from all the sensors, and finally transmits the data to a task manager. If the sensors cannot directly communicate with the sink, some intermediate sensors performs the operation of forwarding the data to sink [1]. Wireless Sensor Networks (WSNs) have emerged as one of the key enablers in recent years for a variety of applications such as environment monitoring, vehicle tracking and mapping, and emergency response. One important problem in such applications is finding the position of a node. To solve the localization problem, it is natural to consider placing sensors manually or equipping each sensor with a GPS receiver. Constraints such as cost and power consumption make these two methods inefficient in the network, especially 
for large-scale WSNs. Hence a variety of approaches have been devised for sensor network localization [2]. Location discovery is emerging as one of the more important tasks as accurate location information could greatly improve the performance of tasks such as routing, energy conservation, data aggregation and maintaining network security $[3,4]$. Localization in wireless sensor networks is performed in following steps. First, distance estimation: this phase involves measurement techniques to estimate the relative distance between nodes. Position computation: it consists of algorithms to calculate the coordinates of the unknown node with respect to the location of known anchor nodes or other neighboring nodes. Localization algorithms require techniques for location estimating depending on the beacon nodes' location. These are called multi-lateration (ML) techniques. Iterative ML: Some nodes may not be in the direct range of three beacons. Once a node estimates its location, it sends out a beacon, which enables some other nodes to now receive atleast three beacons. Iteratively, all nodes in the network can estimate their location but location estimation may not be accurate as errors may propagate. Collaborative MLis when two or more nodes cannot receive atleast three beacons each, they collaborate with each other. Figure 1 shows nodes A and B have three neighbors each. Of the six participating nodes, four are beacons, whose positions are known. Proximity technique is used when there is no range information available. It reveals whether or not a node is in range or near to a reference point.

Localization algorithms using this technique determine if a node is in proximity to a reference point by enabling the reference to transmit periodic beacon signals and whether the node is able to receive at least certain value of the beacon signals set as threshold. In a period, $t$ if it receives $n$ beacons greater than the set threshold then it is in proximity to that reference point $[5,6]$. Localization algorithms: it determines how the information concerning distances and positions, is manipulated in order to allow most or all nodes of WSN to estimate their position. Optimally the localization algorithms may involve algorithms to reduce the errors.

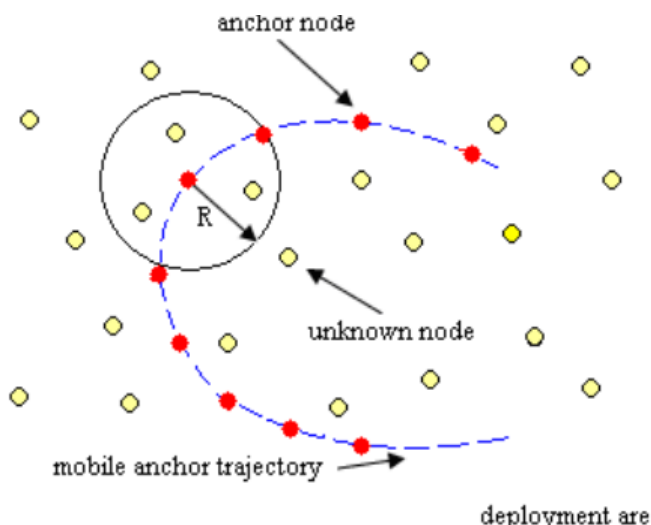

Figure 1. Network architecture

In this paper, we propose a mobile anchor assisted localization algorithm based on Trilateration method (LUMAT) with the objectives of maximizing localization ratio, energy efficiency and localization accuracy. LUMAT uses $\log _{\mathrm{e}}(\mathrm{n})$ number of mobile anchor nodes as the reference nodes, which move in the sensing field and broadcast their current position periodically. Sensor nodes receive the position information of the mobile anchor nodes and localize themselves by using Trilateration algorithm. The results of simulations and measurements show that LUMAT is a practical method that can be used in real-world system, and is also a method of principle simple, less computing and communication, low cost, and high accuracy. The rest of the paper is organized as follows: the next section surveys related works on previous localization research, especially about methods based on mobile localization with more details in order to clarify our work. In section III, we describe the LUMAT method. Section IV reports our simulation and experimental results. Finally, we present our conclusion in section V.

\section{RELATED WORK}

A general survey about localization for wireless sensor networks is found in which is a broad research area in the past several years. A brief survey about various range-free approaches and localization methods which involve mobile reference nodes are provided here. Energy consumption phenomenon has always been noticed in sink-based wireless sensor networks. This paper explores an energy efficient routing protocol with a mobile sink based on the shortest path data transmission mode [7]. According to the position of the sink node and the common nodes' ID in the network, we calculate the coordinate value of each node in the network.

Trilateration based localization method using mobile anchor in wireless sensor networks (M. G. Kavitha) 
By comparing the coordinate values to choose the shortest path to forward the data. Simulation results show that this method can prolong the lifetime of the network, improve the energy utilization ratio and the energy consumption more balanced. Clustering methodology play a significant role in improving the lifetime of sensor network. There has been various hierarchical clustering that has been developed in recent time by enhancing the protocol [8]. The drawback of these protocols is energy of cluster head degrades very fast due to long distance transmission and packet failure likelihood is not considered for inter and intra cluster transmission. To address the energy efficiency issue of existing approach this work proposed packet failure likelihood estimation model and hop selection optimization model for inter cluster transmission.

Positional accuracy is very important indicator for assessing the location of performance. More localization is high precision location of the performance is better [9]. A conclusion might elaborate on the importance of the work or suggest applications and extensions. In addition, the accuracy of the location of the amorphous algorithm is superior to that of other algorithms and there is not a large increase of energy consumption, which is why it is suitable for the location of network nodes large scale. Enhancing the lifetime of the sensor network and at the same time maintaining proper security is an important aspect in wireless sensor networks [10]. In this paper we use the concept of clustered wireless sensor networks. Clustering is a key concept for enhancing the sensor network's lifetime. Cluster wireless sensor networks have mainly two benefits than non clustered WSNs. They are: reducing flow of packets through the network and saving energy by placing unused nodes in sleep mode. Jiang [11] proposed a novel localization approach where unknown nodes through their near anchor nodes to obtain their position. In order to reduce error during localization, a new means was used to approximate the distance between unknown nodes and anchor nodes when it is larger than node's communication radius. Including this, self-adapting genetic algorithm is proposed to calculate the similar position of nodes, it makes the localization error much lower than the common method.

Yetkin and Gungor [12] proposed a new Received Signal Strength Indicator (RSSI) based fingerprint technique which uses logical inferences. Here closed area was divided into the cells of 1 x $1 \mathrm{mt}$. The RSSI characteristics of each cell were recorded into a database in order to prepare a radio map. At real time, the RSSIs of anchor nodes received from base station were compared with radio map according to logical algorithms. In this scheme, the target localization was carried out mathematically. Wei Zhag [13] proposed a two-phase robust localization algorithm based on Consistency of Beacons in Grid. In the first-phase, a voting method based on the consistency of beacons in the grid is used to filter out part of the suspicious nodes. In the second-phase, it was adopted the loss function in M-estimation of Robust Statistics to obtain a robust solution with the remained nodes. Zhang and Hong Pei [14] explored a two-hop Collaborative Multilateral Localization Algorithm (CMLA). This algorithm was implemented through event-driven schemes. It is also introduced a new method which is used to estimate the distances between two hop nodes, applies anchor nodes within two hops to localize unknown nodes, and uses the minimum range error estimation to compute coordinates of unknown nodes. If any unknown node cannot be localized through two hop anchors nodes, it was localized by anchors and localized nodes within two hops.

Chengpei [15] implemented a WSN localization method based on plant growth simulation algorithm (PGSA). This algorithm is a bionic random algorithm that characterizes the growth mechanism of plant phototropism. Based on simulation analysis, this algorithm (PGSA) is simple, fast convergence and robustness, which is more suitable for the large-scale environment. Long Cheng [16] presented a comprehensive analysis of these challenges: localization in non-line-of-sight, node selection criteria for localization in energyconstrained network, scheduling the sensor node to optimize the trade off between localization performance and energy consumption, cooperative node localization, and localization algorithm in heterogeneous network. Including this it was introduced that the evaluation criteria for localization in wireless sensor network. Oguejifor [17] implemented a localization system that uses a RSSI trilateration approach in a wireless sensor network. The system position estimation accuracy was also evaluated. Finally it was concluded that for the proposed system to work there must be the availability of at least three anchor nodes within the network and whenever anchor nodes broadcast packets containing their locations and other sensed parameters, the blind node within the broadcast range can always estimate its distance to the anchor nodes, and if peradventure the blind nodes receive packets from at least three anchors, the blind node can localize its position.

Xiajoun Zhu [18] examined two candidate solutions developed from existing ideas, with one assuming that nodes can hear from each other if and only if they are within transmission range, and the other assuming closer nodes observe larger RSSI. Both candidate solutions do not work well in practice. After changing "closer" to "the closest" and "larger" to "the largest" in the second approach, it was found that the new assumption is quite reliable in practice. Rama and Parvadha [19] proposed a fuzzy logic-based restriction system suitable for remote sensor hubs that are portable in uproarious, savage situations. The constituent frameworks used fuzzy multi lateration and a grid prediction to process the area of a hub as a zone. The signal strength is thrown into bins which encode the imprecision. Laslo [20] presented WSN based fingerprinting localization method. The RSSI values of the communication links between the previously situated sensors and 
the mobile sensor were recorded in an indoor environment through the experiment. Using the recorded RSSI values a feed-forward type of neural network was trained. The result of the training is a neural network capable of performing indoor localization. The accuracy of the localization between the real and the calculated values was measured with Euclidean distance and demonstrated with the cumulative distribution function.

Priti and Tyagi [21] proposed a technique called Multidimensional scaling which computes the position of nodes which are in the communication range of each other. This analysis technique find out the relative position of nodes with accuracy sufficient enough for most of the applications so as to solve the problem of recreation. Martin and Ramalakshmi [22] developed a localization system that carries high-location estimation accuracy at low cost. The system used spatiotemporal properties of well-controlled events in the network; light in this case, to obtain locations of sensor nodes. The system was to detect the multiple events in the network and to increase the area of the sensor field by increasing the number of nodes. By handling this kind of detection of multiple events in the network at once, mainly the time was saved. Sachin Deshpande [23] presented the methodology that gives a solution to compute the state parameters of the adversary target and tracks it and associate the same with the location in the periphery of wireless sensor networks.

Nirmala [24] discussed a new technique that aims to localize all the sensor nodes in the network using trilateration, and a security protocol was used for providing confidentiality and authentication between anchor nodes and sensor nodes. Baihua and Guoli [25] proposed a new method, based on radial distance modulation, to detect and locate moving object from top view angle. This method has advantages of extracting information directly from the moving object characteristics of movement and spatial position, small computation, good robustness, convenient configuration, non-contact etc. It can locate the moving object with simple information after modulating and encoding the perception area of sensors. Dan and Daniel [26] proposed another anchor node localization technique that can be used when GPS devices cannot accomplish their mission or are considered to be too expensive. This novel technique was based on the fusion of video and compass data acquired by the anchor nodes and is especially suitable for video- or multimedia-based wireless sensor networks. Divya [27] proposed a mobility control scheme and we explored the impact of mobility over the performance of wireless sensor network. Two different protocols were used for the performance analysis of proposed mobility control scheme and the impact of this method over the selected protocols. It was analyzed the performance of the protocols on the basis of different parameters like Throughput, Packet Delivery Ratio, Routing Load and energy consumption. Jang Ping Sheu [28] proposed distributed localization scheme where each normal node gathers the necessary information via two-hop flooding and is thus scalable. Aside from this, each normal node uses a simplified approach and the proposed improved grid-scan algorithm to find the initial estimated locations of the normal node, thus reducing the computation cost. It also introduced a vector-based refinement scheme to correct the initial estimated location of the normal node, thus improving the accuracy of the estimated location.

The nodes which are aware of their locations using special positioning devices are called anchor nodes or reference nodes. Other nodes that do not initially know their locations are called unknown nodes or sensor nodes. Generally, an unknown node estimates its location by range-based or range-free methods if three or more anchors are available in its 2-dimensional coverage field [29]. In all these better localization precision is achieved with the increased number of anchor nodes. The main problem with the increased number of anchors is that they are far more expensive than the rest of the sensors. The price of the whole network will increase even if only $10 \%$ of the nodes are anchor nodes. After the unknown/ stationary nodes have been localized, the anchors become useless. For this reason, it is necessary to consider an optimal number of mobile anchors to localize the sensor network [30].

The main idea of localization using a mobile anchor node is as follows a mobile anchor node traverses the sensor network by broadcasting anchor packets that contain the coordinates of the anchor node after the deployment of sensor network. Sensor nodes that receive anchor packets possibly will infer their distance from a mobile anchor node and use these measurements as constraints to construct and maintain position estimates. These methods have a common feature such as they use range-based approaches.Based on these analyses, localization using an optimal number of mobile anchor nodes would be more economy. In addition, it is necessary to consider the constraints in computing and memory power of sensors with an optimal number of mobile anchors for efficient localization in wireless sensor networks.

\section{RESEARCH METHOD}

\subsection{System environment}

In the simulation, the considered WSN consists of two types of sensors including static sensor nodes and $\log _{\mathrm{e}}(\mathrm{n})$ number of mobile anchor nodes. Static sensor nodes are randomly deployed in a two-dimensional coordinate system. The locations of static sensors are unknown since they do not have GPS facility. The mobile anchor nodes are equipped with GPS receiver to determine their locations when they navigate over the sensing 
region. The anchor nodes which are mobile in nature and sensors are able to receive messages from these anchors in the sensing region.

\subsection{Beacon messages}

In the proposed model the anchor nodes periodically send message packets containing their position coordinates while navigating in the sensing area. These messages are called beacon messages. When an unknown sensor node receives at least three such message packets, it calculates its position coordinates using trilateration method. We assume that the distance between the mobile anchor nodes, any unknown node is estimated using RSSI technique.

\subsection{Energy model}

The mobile anchor has sufficient initial energy for moving and broadcasting anchor packets during localization is the basic assumption we consider regarding energy level. Number of bits transmitted in a message and distance travelled affect the energy level of anchor nodes.

\subsection{LUMAT Method}

\subsubsection{Network segmentation}

Assume the sensing region is divided into several hexagons. The mobile anchor nodes traverse the entire region in order to cover all sensor nodes. Since the sensing region is in irregular shape some of the regions may not be covered by hexagonal space.

\subsubsection{Mobile anchor node}

The anchor nodes randomly traverse around the entire network, which periodically broadcast messages. The mobile anchor traverses the entire region at the speed $\mathrm{V}$ and broadcasts its current location $\left(\mathrm{x}_{\mathrm{i}}, \mathrm{y}_{\mathrm{i}}\right)$ with an interval $\mathrm{L}$ and a communication range $r$ as depicted in Figure 2. The pseudo code for mobile anchor is described below.

Input: $\{(\mathrm{x}, \mathrm{y})-$ coordinates of anchor nodes, L-interval $\}$

Output: M-message

Process:

Set initial timer $=0$

// broadcasting positions with an interval $\mathrm{L}$

If (timer $\% \mathrm{~T}=0$ ) then

$\left(\mathrm{x}_{\mathrm{i}}, \mathrm{y}_{\mathrm{i}}\right)=\operatorname{GetPosition}(\mathrm{xi}, \mathrm{yi})$

Msg $=$ MakeMessage $\left(\mathrm{x}_{\mathrm{i}}, \mathrm{y}_{\mathrm{i}}\right)$

Broadcast (msg);

End if

\subsubsection{Unknown nodes}

Unknown nodes receive positions of the mobile anchor nodes continuously, and save the coordinates within a certain period of time " $T$ ", i.e. the time that mobile anchor nodes complete all information broadcasting in the sensing region. If a node receives several messages at a time it considers the messages having higher signal strength and continues finding its location. Trilateration method is used to estimate positions $\left(x_{\text {esst }}, y_{\text {est }}\right)$ of unknown nodes.

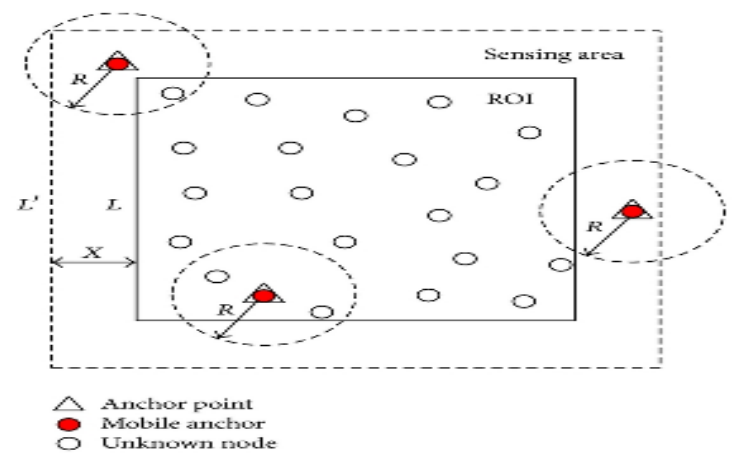

Figure 2. System environment with mobile anchor nodes 
The following is the pseudo code for unknown nodes

Input: $\mathrm{T}$ - time period, $\mathrm{M}$ - message

Output: ( $\left.\mathrm{x}_{\mathrm{est}}, \mathrm{y}_{\mathrm{est}}\right)$

Process:

Set number of received messages $n=0$

Set clock initialization timer $=0$

// receive messages continuously within a time period of $\mathrm{T}$

\{

While (timer! $=\mathrm{T}$ )

msg $=$ ReceiveMessage ()

$\mathrm{n}++$

\}

RecordPosition (n, msg)

Calculate $\left(\mathrm{x}_{\mathrm{est}}, \mathrm{y}_{\mathrm{est}}\right)$

\subsubsection{Trilateration}

An example of the trilateration is shown in Figure 3. Unknown node $D(x, y)$ receives three anchor packets from the mobile anchor, namely, $A\left(x_{a}, y_{a}\right), B\left(x_{b}, y_{b}\right)$, and $C\left(x_{c}, y_{c}\right)$. Distances between $A, B, C$, and $D$ are $d_{a}, d_{b}$, and $d_{c}$ respectively. Since the unknown node $D$ is within the regular triangle which is composed of $A, B$, and $C$, unknown node $D$ will calculate its location by using:

$$
\begin{aligned}
& \left(-x_{a}\right)^{2}+\left(y-y_{a}\right)^{2}=d_{a}{ }^{2} \\
& \left(-x_{b}\right)^{2}+\left(y-y_{b}\right)^{2}=d_{b}{ }^{2} \\
& \left(-x_{c}\right)^{2}+\left(y-y_{c}\right)^{2}=d_{c}{ }^{2}
\end{aligned}
$$

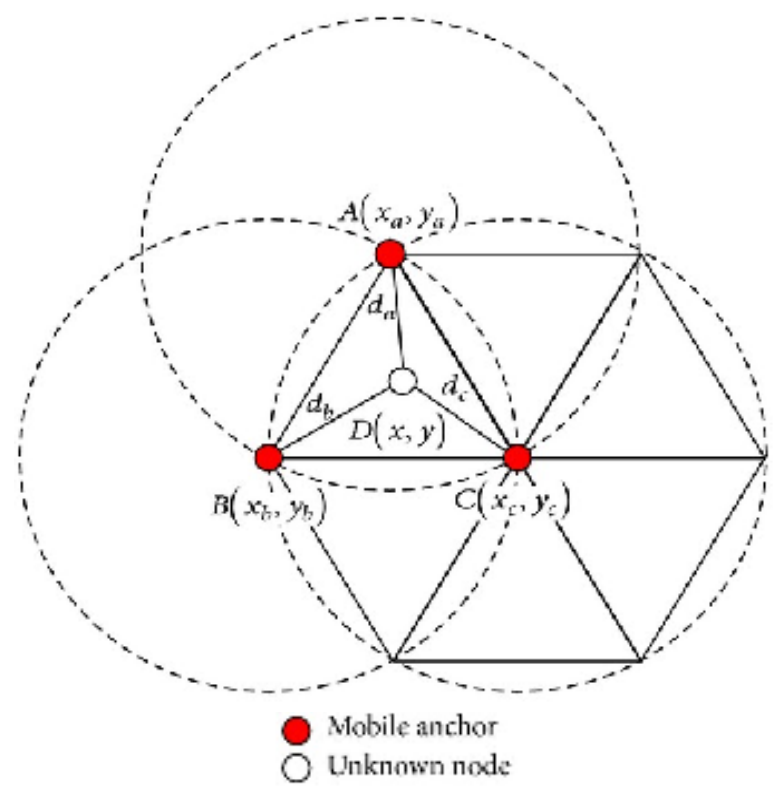

Figure 3. Trilateration based localization

\section{RESULTS AND ANALYSIS}

\subsection{Evaluation criteria}

a. Localization Ratio

Localization ratio is the ratio of the number of unknown nodes localized to the total number of unknown nodes. This metric also indicates the coverage degree of the movement path. Localization ratio is defined as

$$
\mathrm{L}_{\text {ratio }}=\mathrm{N}_{\mathrm{l}} / \mathrm{N}_{\mathrm{u}}
$$


Where $N_{l}$ is the number of localizable unknown nodes and $N_{o}$ is the total number of unknown nodes.

b. Localization Accuracy

The localization error of unknown node $u$ is defined as

$$
\mathrm{e}_{\mathrm{u}}=\underline{\sqrt{\left(\mathrm{x}_{\mathrm{u}}-\mathrm{a}_{\mathrm{u}}\right)^{2}+\left(\mathrm{y}_{\mathrm{u}}-\mathrm{b}_{\mathrm{u}}\right)^{2}+\left(\mathrm{z}_{\mathrm{u}}-\mathrm{c}_{\mathrm{u}}\right)^{2}}} \mathbf{R}
$$

Where $\left(\mathrm{x}_{\mathrm{u}}, \mathrm{y}_{\mathrm{u}}, \mathrm{z}_{\mathrm{u}}\right)$ are real coordinates of an unknown node $\mathrm{u},\left(\mathrm{a}_{\mathrm{u}}, \mathrm{bu}, \mathrm{cu}\right)$ are estimated coordinates of an unknown node $\mathrm{u}$, and $\mathrm{R}$ is the communication range of sensor nodes.

c. Path Length

To save energy consumption and time for localization, the path length of the mobile anchor node should be as short as possible.

d. Scalability

Scalability means that the localization performance is independent of the unknown nodes density.

\subsection{Simulations and analysis}

Hundred sensor nodes are randomly deployed in a $100 \mathrm{~m} \times 100 \mathrm{~m}$ square region as shown in Figure 4 . Each sensor can communicate with the mobile anchor nodes if distance between them is smaller than sensor range R. Figure 5 to Figure 7 show the localization error ratio, energy consumption of nodes and the coverage ratio localization.

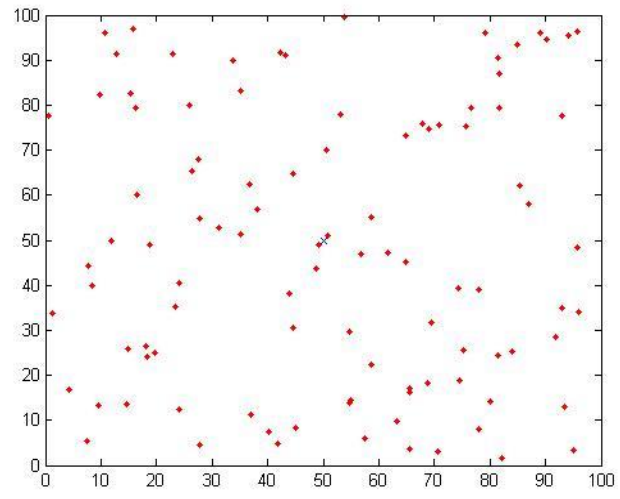

Figure 4. Random distribution of nodes

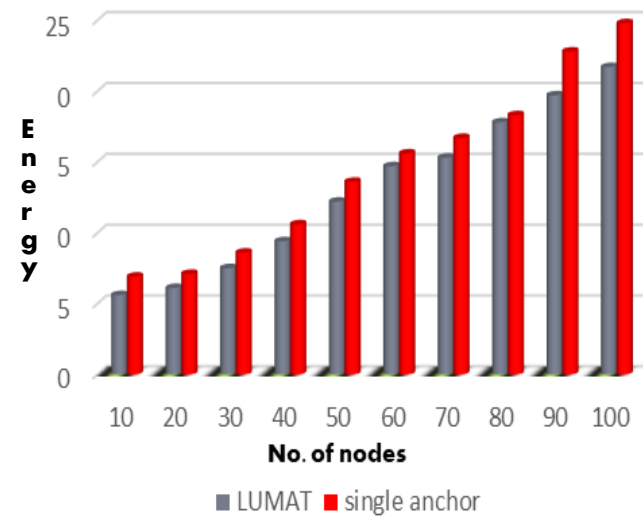

Figure 6. Energy consumption of nodes

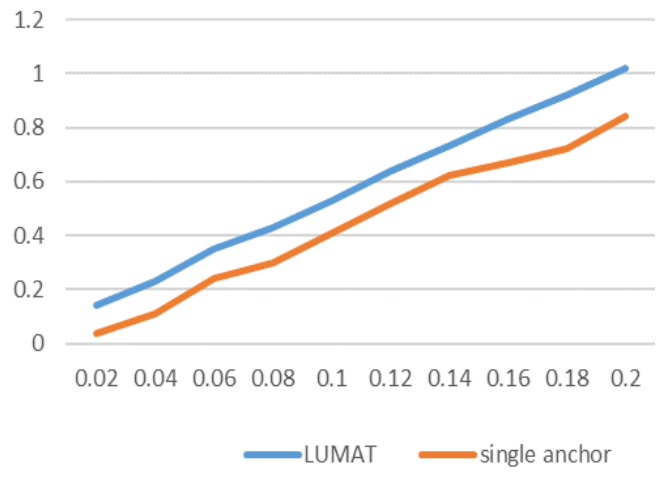

Figure 5. Localization error ratio

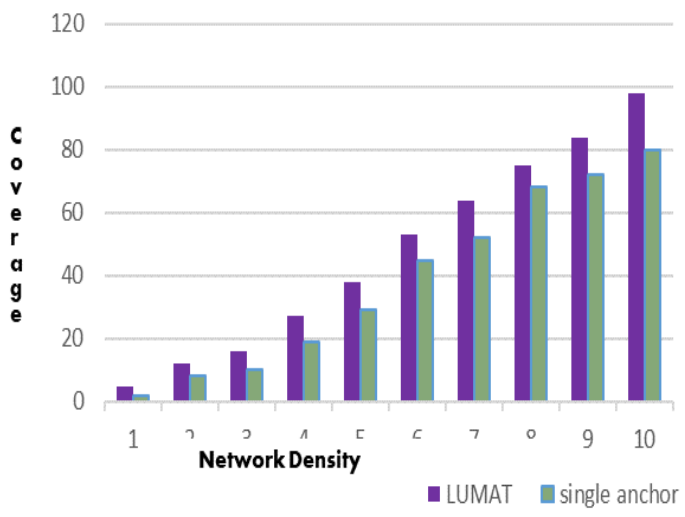

Figure 7. Coverage ratio during localization 
The key metric for evaluating a localization technique is the accuracy of the location estimates versus the energy consumption and localization ratio. Localization accuracy mainly depends on increased density of anchors or number of the broadcasting messages, but the tradeoffs need to be made to determine appropriate deployment parameters. Table 1 presents the performance analysis.

\begin{tabular}{ccccccc}
\hline Metrics & $\begin{array}{c}\text { Detection } \\
\text { efficiency }(\%)\end{array}$ & $\begin{array}{c}\text { PDR } \\
(\mathrm{pkts})\end{array}$ & $\begin{array}{c}\text { Network L } \\
\text { time (Secs) }\end{array}$ & $\begin{array}{c}\text { End to end } \\
\text { delay }(\mathrm{msec})\end{array}$ & $\begin{array}{c}\text { Energy } \\
\text { consumption \% }\end{array}$ & $\begin{array}{c}\text { Packet Integrity } \\
\text { Vs Speed }\end{array}$ \\
\hline LUMAT & $65-99$ & $23-93$ & $456-987$ & $0.32-0.11$ & $11-19 \%$ & $12-25$ \\
SINGLE & $47-68$ & $18-55$ & $256-687$ & $0.562-0.315$ & $29-32 \%$ & $76-52$ \\
ANCHOR & & & & & & \\
\hline
\end{tabular}

\section{CONCLUSION}

In this paper we propose how to localize individual nodes in a network when the anchors are mobile in nature. An optimal number of anchors are considered for efficient localization of nodes in the network. Simulations and tests show that proposed localization method is energy efficient and accurate in nature. Also the ratio of coverage of nodes in the network is high. Simulation results show that our LUMAT algorithm significantly outperforms the localization method containing single anchor node in the network. Anchors should be designed dynamically or partially according to the observable environment or deployment situations to make full use of real-time information during localization. This is the future research issue in the area of mobile anchor assisted localization algorithm.

\section{REFERENCES}

[1] Z. Hu, D. Gu, Z. Song, and H. Li, "Localization in Wireless Sensor Networks Using a Mobile Anchor Node," Proceedings of the 2008 IEEE/ASME, International Conference on Advanced Intelligent Mechatronics, 2008.

[2] K.F. Ssu, C.H. Ou, and H. C. Jiau, "Localization with mobile anchor points in wireless sensor networks," IEEE Trans. on Vehicular Technology, vol. 54, no. 3, pp. 1187-1197, 2005.

[3] Yurong Xu, Yi Ouyang, Zhengyi Le, James Ford, and Fillia Makedon, "Mobile Anchor-free Localization for Wireless Sensor Networks," International Conference on Advanced Intelligents, 2007.

[4] Jinfang Jiang, Guangjie Han, Huihui Xu, Lei Shu, and Mohsen Guizani, "LMAT: Localization with a Mobile Anchor node based on Trilateration in Wireless Sensor Networks," IEEE Globecom proceedings, 2011.

[5] Sayyed Majid Mazinani and Fatemeh Farnia, "Localization in Wireless Sensor Network Using a Mobile Anchor in Obstacle Environment," International Journal of Computer and Communication Engineering, vol. 2, no. 4, 2013.

[6] Kuo-Feng Ssu, Chia-Ho Ou, and Hewijin Christine Jiau, "Localization With Mobile Anchor Points in Wireless Sesnor Networks," International Journal of Computer Communications, vol. 8, no. 6, 2015.

[7] Ming Chen, Xumin Xu, Shaohui Zhang, and Guofu Feng, "Energy Efficient Routing Protocol in Mobile-Sink Wireless Sensor Networks," TELKOMNIKA (Telecommunication Computing Electronics and Control), vol. 10, no. 8, pp. 2056-2062, 2012.

[8] Madhu Patil and Chirag Sharma, "Energy Efficient WSN by Optimizing the Packet Failure in Network," Indonesian Journal of Electrical Engineering and Computer Science, vol. 7, no. 2, pp. 415-425, 2017.

[9] R. Khadim, M. Erritali, and A. Maaden, "Rang-Free Localization Schemes for Wireless Sensor Networks," TELKOMNIKA (Telecommunication Computing Electronics and Control), vol. 16, no. 2, pp. 323-332, 2015.

[10] M. Ali Hussain, "Energy Efficient Intrusion Detection Scheme with Clustering for Wireless Sensor Networks," TELKOMNIKA (Telecommunication Computing Electronics and Control), vol. 15, no. 1, pp. 128-141, 2015.

[11] N. Jiang, S. Jin, Y. Guo, and Y. He, "Localization of Wireless Sensor Network Based on Genetic Algorithm," International Journal of Computer Communications, vol. 8, no. 6, pp. 825-837, 2013.

[12] Yetkin Tatar and Gungor Yildirim, "An Alternative Indoor Localization Technique Based on Fingerprint in Wireless Sensor Networks," International Journal of Advanced Research in Computer and Communication Engineering, vol. 2, no. 2, pp.1288-1294, 2013.

[13] Wei Zhang, Wenqing Liu, Yunfang Chen, and Zeyu Ni, "Robust Secure Localization of WSN Based on Consistency of Beacons in Grid," Journal of Computational Information Systems, vol. 10, no. 6, pp. 2283-2295, 2014.

[14] Shaoping Zhang and Hong Pei, "A Two-hop Collaborative Localization Algorithm for Wireless Sensor Networks," TELKOMNIKA (Telecommunication Computing Electronics and Control), vol. 11, no.5, pp. 2432-2441, 2013.

[15] C. Tang, R.i Liu, and J. Ni, "A Novel Wireless Sensor Network Localization Approach: Localization based on Plant Growth Simulation algorithm" Elektronika IR Elektrotechnika, vol. 9, no. 8, pp. 97-100, 2013.

[16] Long Cheng, Chengdong Wu, Yunzhou Zhang, HaoWu, Mengxin Li, and Carsten Maple, "A Survey of Localization in Wireless Sensor Network," International Journal of Distributed Sensor Networks, pp.1-13, 2012.

[17] O.S. Oguejiofor, V.N. Okorogu, Adewale Abe, B. Osuesu, "Outdoor Localization System Using RSSI Measurement of Wireless Sensor Network," International Journal of Innovative Technology and Exploring Engineering, vol. 2, no. 2, pp. 1-6, 2013. 
[18] Xiaojun Zhu, Xiaobing Wu, and Guihai Chen, "Relative localization for wireless sensor networks with linear topology," Elsevier, vol. 36, pp. 1581-1591, 2013.

[19] M. Rama Prabha and R. Parvadha Devi, "Efficient Node Localization in Mobile Wireless Sensor Network," International Journal of Advanced Research in Computer Science \& Technology, vol. 2, no. 1, pp. 246-249, 2014.

[20] Laslo Gogolak, Szilveszter Pletl, and Dragan Kukolj, "Neural Network-based Indoor Localization in WSN Environments," Acta Polytechnica Hungarica, vol. 10, no. 6, pp. 221-235, 2013.

[21] Priti Narwal and S.S. Tyagi, "Position Estimation using Localization Technique in Wireless Sensor Networks," International Journal of Application or Innovation in Engineering \& Management, vol. 2, no. 6, pp. 110-115, 2013.

[22] M. Victor and K. Ramalakshmi, "Multiple Event-Driven Node Localization in Wireless Sensor Networks," Int. J. of Advanced Research in Computer Engineering \& Technology, vol. 2, no. 3, pp.1073-1077, 2013.

[23] Sachin Deshpande, Umesh Kulkarni and Mritunjaykumar Ojha, "Target Tracking inWireless Sensor Network," International Journal of Emerging Technology and Advanced Engineering, vol. 3, no. 9, pp. 177-181, 2013.

[24] M. B. Nirmala, Nayana, and A .S. Manjunath, "Localization of Wireless Sensor Networks Using Robust Estimated Trust Evaluation Model", Int. Journal of Scientific Engineering and Technology, vol. 2, no. 7, pp.729-732, 2013.

[25] Baihua Shen and Guoli Wang, "Distributed Target Localization and Tracking with Wireless Pyroelectric Sensor Networks," International Journal on Smart Sensing and Intelligent Systems, vol. 6, no. 4, pp. 1400-1418, 2013.

[26] Dan Pescaru and Daniel-Ioan Curiac, "Anchor Node Localization for Wireless Sensor Networks Using Video and Compass Information Fusion,” Sensors, vol. 14, pp. 4211-4224, 2014.

[27] Divya Bharti, Manjeet Behniwal, and Ajay Kumar Sharma, "Performance Analysis and Mobility Management in Wireless Sensor Network," International Journal of Advanced Research in Computer Science and Software Engineering, vol. 3, no. 7, pp. 1333-1342, 2013.

[28] Jang-Ping Sheu, Pei-Chun Chen, and Chih-Shun Hsu, "A Distributed Localization Scheme for Wireless Sensor Networks with Improved Grid-Scan and Vector-Based Refinement," IEEE Transactions on Mobile Computing, vol. 7, no. 9, pp. 1110-1123, 2008.

[29] K. Vinoth Kumar and S. Bhavani, "Localization based Optimized Energy Routing for Wireless Sensor Networks," Middle East Journal of Scientific Research (MEJSR), vol. 23, no. 05, 2015

[30] K. Vinoth Kumar, T. Jayasankar, V. Srinivasan, and M. Prabhakaran, "EOMRP: Energy Optimized Multipath Routing Protocol for Wireless Sensor Networks," International Journal of Printing, Packaging \& Allied Sciences, vol. 4, no. 1, pp. 336-343, 2016. 